Printed in Great Britain

\title{
Ploidal Inheritance in the Slime Mould Dictyostelium discoideum: Haploidization and Genetic Segregation of Diploid Strains
}

\author{
By RAQUEL R. SUSSMAN AND M. SUSSMAN \\ Brandeis University, Waltham, Massachusetts, U.S.A.
}

(Received 14 May 1962)

\begin{abstract}
SUMMARY
Diploid and metastable strains of Dictyostelium discoideum were cultivated in liquid medium for extended periods of exponential growth. A progressive increase occurred in the proportion of cells yielding stable haploid clones. The haploidization of a doubly heterozygous diploid yielded the expected segregant classes.
\end{abstract}

\section{INTRODUCTION}

Dictyostelium discoideum is a cellular slime mould. The extraordinary capacity of this group for multicellular organization has been studied by many workers. The development of a suitable chromosome staining procedure (Wilson, 1953; Wilson \& Ross, 1955; Ross, 1960; Sussman, R.R. 1961) has focused interest on its cytogenetics. Examination of mitotic figures revealed the existence of haploid and diploid amoebae (Wilson \& Ross, 1957; Ross, 1960) and the ploidal composition of a culture was found to be stable and clonally inherited (Ross, 1960; Sussman \& Sussman, 1962). Three general types of strains have been encountered so far: (i) stable haploid, containing a negligible proportion of diploid derivatives; (ii) stable diploid, containing a negligible proportion of haploid derivatives; (iii) metastable strains containing appreciable proportions of both. The ploidal compositions of these strains are maintained during serial subcultures and clonal re-isolations. Properties of the three types of strains, including size distributions of spores and myxamoebae and certain morphogenetic capacities, were described by Sussman \& Sussman (1962). They also specified some conditions under which each of the types could be isolated from the others. The present paper is concerned with a more detailed examination of the conditions of cultivation which permit the haploidization of stable diploid and metastable strains.

\section{METHODS}

Organisms. Two stable diploid strains of Dictyostelium discoideum, RA and $\mathrm{H}-1$, and one metastable stock, I-2 A, were used.

Cultivation. Mass and clonal cultures were maintained on SM agar in association with Aerobacter aerogenes (Sussman, 1951). Many of the growth studies were made in liquid media of two kinds, the first being a suspension of washed $A$. aerogenes in M/60 phosphate buffer (Gerisch, 1960), the second a growing culture of $A$. aerogenes in a nutrient broth (Sussman, 1961 a). Twenty ml. volumes in Erlenmeyer flasks

Vol. 30, No. 2 was issued 25 February 1963 
were shaken ( 200 cycles/min. with a 1.5 in. stroke) at $22^{\circ}$. Growth was measured by total and viable cell counts.

Size distributions of spores. Spores from haploid strains of Dictyostelium discoideum are regular ellipsoids. Spores from diploid strains are much more irregular and often banana, boomerang or serpentine in shape. In both cases, the major diameter is an accurate measure of cell size (Sussman \& Sussman, 1962). Sizing was done with wet mounts under an oil-immersion objective, with an ocular micrometer. Spore lengths are given as micrometer divisions ( 1 division $=\mathbf{0} \cdot 83 \mu$ ).

Chromosome counts. The staining procedure involves fixation in Carnoy's fluid and exposure to aceto-orcein after acid hydrolysis. Metaphase chromosomes are immediately and clearly visible under phase contrast and to some extent under bright field. (See Sussman, R. R. 1961, for photomicrographs.)

\section{RESULTS}

\section{Criteria used to detect ploidal composition}

The fundamental criterion by which the ploidal varieties have been defined is, of course, the number of chromosomes viewed in metaphase. The haploid number in Dictyostelium discoideum is 7. Thus, of several hundreds of countable figures in strains RA and H-1, none was haploid, while haploid stocks derived later on from these showed no diploid metaphase figures out of several hundred examined. Strain I-2 A has on all occasions shown a preponderance $(c .90 \%)$ of diploid chromosome sets. Other metastable strains display a preponderance of haploid cells (Sussman \& Sussman, 1962).

The size distributions of both spores and myxamoebae could be strictly correlated with ploidy (Sussman \& Sussman, 1962). This correlation made it possible to diagnose the ploidy of a clone by microscopic inspection of its spores. The criteria used are: $(a)$ the incidence of spores big enough $\left(d_{m} \geqslant 16\right.$ div.) or small enough $\left(d_{m} \leqslant 8 \mathrm{div}\right.$.) to be at the high extreme of the diploid or the low extreme of the haploid size distributions; $(b)$ the mean major diameters of the populations; $(c)$ the spore shapes. Many spot checks made by inspection of mitotic figures in the course of this and a previous investigation (Sussman \& Sussman, 1962) support the validity of this method of diagnosing ploidy.

\section{The haploidization of strain RA}

Twenty ml. of nutrient broth inoculated with RA myxamoebae at an initial density of $10^{4}$ cells $/ \mathrm{ml}$. and $1 \mathrm{ml}$. of a $24 \mathrm{hr}$. bacterial broth culture were incubated with shaking at $22^{\circ}$. Plate-grown cells experience a lag, but liquid-grown amoebae transferred from the log phase do not. Figure 1 shows the growth kinetics of two such cultures maintained in continuous exponential growth for $\mathbf{4 7}$ and 40 generations respectively by serial passage. The generation time was initially about $4.0 \mathrm{hr}$. but speeded up to $\mathbf{3 \cdot 3} \mathrm{hr}$. after several passages. Samples were plated at intervals in replicate to yield clones. The ploidal compositions of these were scored by spore size determinations and occasionally by chromosome counts. Early samples yielded nothing but diploid clones, but later samples gave rise to progressively increasing proportions of haploid clones. Figure 2 is a summary of data for the two cultures mentioned previously. These are from a total of four experiments and show between 
them the greatest variation. In one, haploids were detected after only 8 generations, and by $\mathbf{3 0}$ generations diploids were undetectable. In the other, haploid clones were not observed until after 20 generations and diploid clones were still found after $\mathbf{4 5}$ generations. In the four experiments, all of the clones examined were characteristic of either the stable haplophase or diplophase on the basis of spore size. Figure 3 shows the size distributions of spores from 10 diploid and from 16 haploid clones chosen at random from one of the experimental plates. They resemble the distributions previously obtained for diploid and haploid stocks.

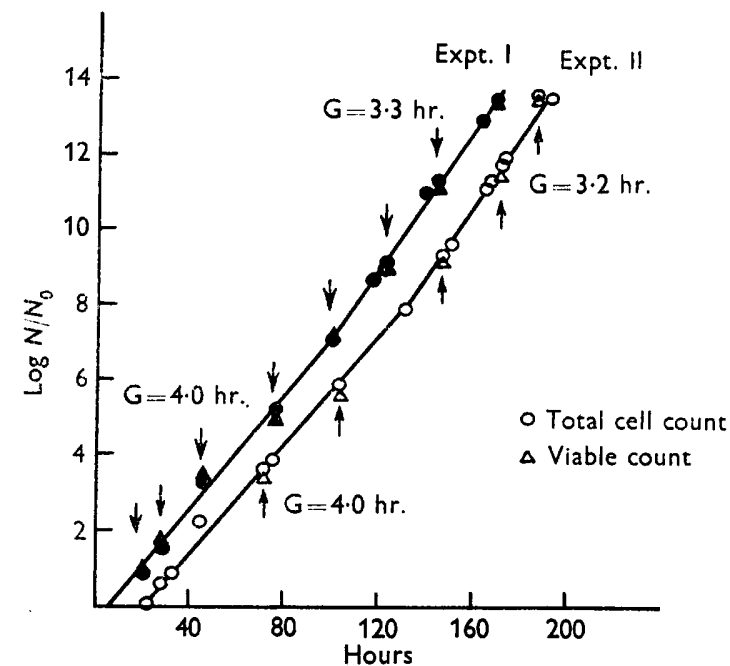

Fig. 1

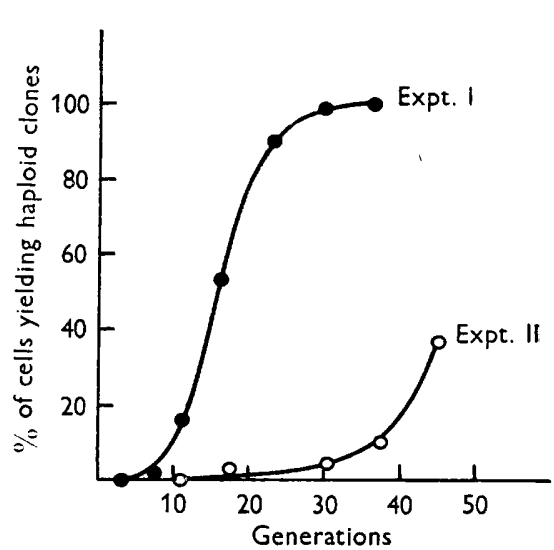

Fig. 2

Fig. 1. Growth kinetics of Dictyostelium discoideum, strain RA. 'G' refers to generation times. The arrows designate the times at which samples were withdrawn for plating in order to score ploidal composition.

Fig. 2. Appearance of cells capable of yielding haploid clones in cultures of Dictyostelium discoideum strain RA. Clones were scored for spore size and chromosome counts in samples taken at the times designated by the arrows in Fig. 1.

\section{Selective advantage of the haploid segregants of strain RA}

In both of the curves shown in Fig. 1, a significant increase in growth rate occurred during the period of cultivation. This suggested that the shift from diplophase to haplophase might have been partly caused by haploid segregants growing faster than their diploid progenitors. Several of the haploid clones to which strain RA has given rise during the sustained exponential growth in liquid culture were replated to ensure clonal homogeneity and then grown in liquid medium in the same manner as described above. All grew significantly faster than the parental RA stock. Figure 4 summarizes the data for one isolate, strain 44-14. A reconstitution experiment was made to see whether the faster growth rate of the haploid segregants could account for the ploidal shift observed in Fig. 2. Two liquid cultures were started, one containing strain RA only; and the other a mixture of RA and 44-14 initially in a ratio of $86: 14$. Samples were plated for clonal analysis of spore size at intervals. during sustained exponential growth. Figure 5 shows that in the control culture, cells capable of giving rise to haploid clones were detected in the controls after 18 
generations and reached about $40 \%$ of the total by 33 generations. Therefore, if the haploid segregant grew at the same rate as its diploid parent when mixed with it, the experimental culture which contained $14 \%$ haploids at zero time would have been expected to yield the same curve as the control but displaced upward from it by $14 \%$ (the lower dashed line in Fig. 5). If, on the other hand, the haploid segregant could grow faster than its diploid parent when mixed with it, one would have expected the proportion of haploids in the experimental culture to increase very much more steeply than in the control. If in fact the difference in growth rates between strains 44-14 and RA were the same, as shown in Fig. 4, one would predict a curve like that given by the upper dashed line. As seen in Fig. 5, the experimental points do fall very near the latter curve.

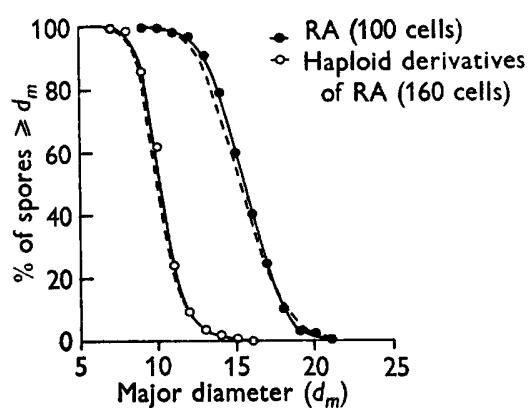

A

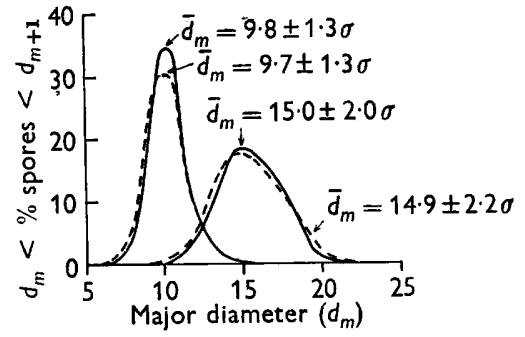

B

Fig. 3. Clones from Expt. I of Fig. 2 were scored for ploidy by chromosome strains. The major diameters of randomly chosen spores from these clones were then measured. The integral curves in A show the percentage of spores whose major diameters were equal to or greater than the values given in the abscissa. The differential curves in $B$ were derived from the upper and show the percentage of spores falling between unit increments in major diameters. The dotted lines are curves previously obtained for RA, a diploid stock and NC-4, a stable haploid stock (Sussman \& Sussman, 1962).

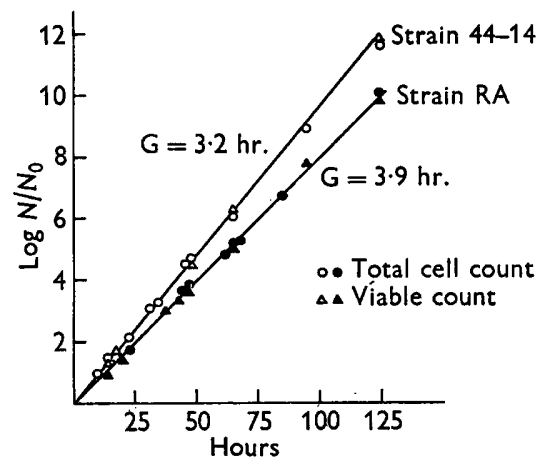

Fig. 4. Growth curves of Dictyostelium discoideum, strain RA and its haploid derivative, 44-14.

Haploidization and genetic segregation in a heterozygous diploid

Strain $\mathrm{H}-1$ is a stable diploid which arose as a single clone from a mixed culture of two metastable mutant strains. The first, I-47, has fruiting bodies with yellow (wild-type) pigment plus a second reddish brown soluble pigment which stains both 
the fruiting bodies and underlying agar. The second, I-262, remains pure white. $\mathrm{H}-1$ is diploid and displays only the yellow pigment but a small $(0 \cdot 1-1)$ percentage of its clones are haploid and display pigmentation patterns corresponding to the four segregant classes (i.e. yellow-brown, white, white-brown, and yellow (Sussman, $1961 b)$ ). The haploid segregants breed true both for pigmentation and ploidy whereas the diploid clones continue to segregate at low frequency.

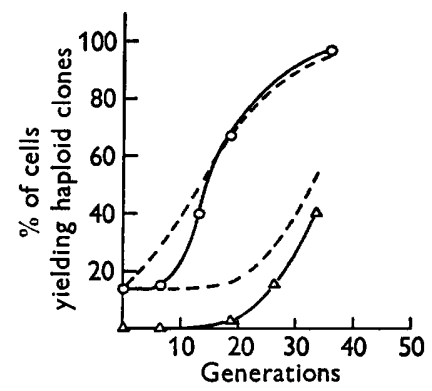

Fig. 5. Haploidization in Dictyostelium discoideum, strain RA $(\triangle)$ and a mixture of RA and 44-14 (O). The lower dashed curve would be expected for the mixture if RA and 44-14 grew at the same rate. The upper dashed curve would be expected if they grew at the rates deduced from Fig. 4.

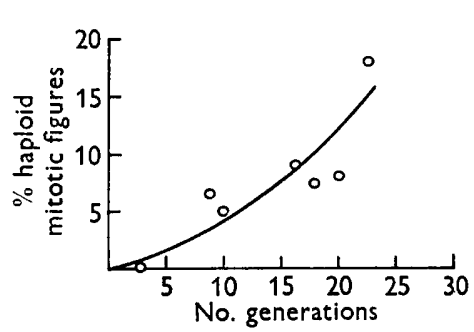

A

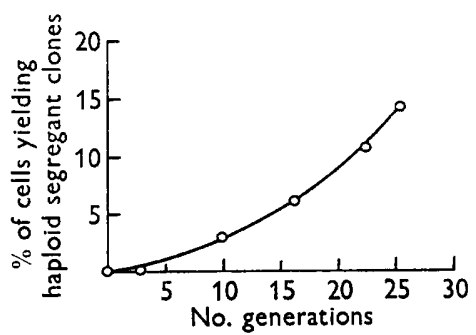

B

Fig. 6. A. the increase in haploid mitotic figures in samples taken from a growing culture of $\mathrm{H}-1$. B. the increase in cells yielding haploid, segregant clones.

Table 1. Haploid segregants from Strain $\mathbf{H}-1$ during sustained exponential growth

\begin{tabular}{|c|c|c|c|c|c|c|c|}
\hline \multirow[b]{2}{*}{$\begin{array}{c}\text { No. of } \\
\text { generations }\end{array}$} & \multirow{2}{*}{$\begin{array}{l}\text { No. of } \\
\text { clones } \\
\text { examined }\end{array}$} & \multirow[b]{2}{*}{$\begin{array}{c}\text { No. } \\
\text { haploid }\end{array}$} & \multicolumn{4}{|c|}{ Pigment phenotypes } & \multirow{2}{*}{$\begin{array}{c}\% \text { of } \\
\text { total } \\
\text { clones }\end{array}$} \\
\hline & & & White & $\begin{array}{l}\text { Yellow- } \\
\text { brown }\end{array}$ & $\begin{array}{l}\text { White- } \\
\text { brown }\end{array}$ & Yellow & \\
\hline $2 \cdot 5$ & 72 & $\mathbf{0}$ & $\mathbf{0}$ & 0 & $\mathbf{0}$ & $\mathbf{0}$ & $\mathbf{0}$ \\
\hline 10 & 64 & 2 & $\mathbf{1}$ & 1 & $\mathbf{0}$ & $\mathbf{0}$ & $\mathbf{3}$ \\
\hline 16 & 72 & 4 & 2 & 1 & $\mathbf{0}$ & 1 & 5.7 \\
\hline 22.5 & 65 & 7 & $\mathbf{2}$ & $\mathbf{3}$ & 1 & $\mathbf{1}$ & $10 \cdot 8$ \\
\hline 26 & 83 & 12 & 4 & $\mathbf{5}$ & 1 & 2 & $14 \cdot 4$ \\
\hline
\end{tabular}

A liquid nutrient culture of $\mathbf{H}-1$ was maintained in the exponential phase for $\mathbf{3 0}$ generations. Samples were withdrawn at intervals for staining. The incidence of haploid mitotic figures rose from an undetectable level to $18 \%$ during 23 generations (Fig. 6A). Samples were also plated and randomly chosen clones scored for ploidy and incubated further to determine pigmentation. The incidence of haploid clones rose from a negligible level to $14 \%$ after 26 generations (Fig. 6B). All clones deter- 
mined to be diploid retained the wild-type pigmentation. All those first determined to be haploid, on subsequent incubation fell into one or another of the four segregant classes described above (Table 1 ).

Cytological observation also revealed, in the later samples, an appreciable incidence of tripolar and tetrapolar mitotic figures. An example of the former in telophase is shown in Pl. 1. A considerable number of binucleate cells was also detected. Strain RA also displayed these anomalies.

\section{Haploidization of metastable strains}

Table 2 summarizes the results of an experiment with strain I-2 A. After 23 generations of sustained exponential growth, the incidence of haploid mitotic figures rose slightly and the incidence of cells that yielded haploid clones increased from a negligible initial level to $6 \%$. Another metastable strain, $1-47$, in which the majority of cells are haploid, yielded a large proportion of haploid clones after only 10 generations.

Table 2. Haploidization of strain $\mathbf{I}-\mathbf{2} \mathrm{A}$

A. Incidence of haploid and diploid mitotic figures

$\begin{array}{cccc}\begin{array}{c}\text { No. of } \\ \text { generations }\end{array} & \begin{array}{c}\text { No. } \\ \text { diploid }\end{array} & \begin{array}{c}\text { No. } \\ \text { haploid }\end{array} & \begin{array}{c}\% \\ \text { haploid }\end{array} \\ 11 & 18 & \mathbf{3} & 14 \\ 17 & \mathbf{2 1} & \mathbf{2} & 9 \\ \mathbf{2 3} & \mathbf{3 4} & \mathbf{9} & \mathbf{2 1}\end{array}$

B. Incidence of stable haploid clones

$\begin{array}{cccc}\begin{array}{c}\text { No. of } \\ \text { generations }\end{array} & \begin{array}{c}\text { No. clones } \\ \text { examined }\end{array} & \begin{array}{c}\text { No. } \\ \text { haploid }\end{array} & \begin{array}{c}\% \\ \text { haploid }\end{array} \\ \mathbf{3 \cdot 8} & 50 & 0 & 0 \\ 17 & 51 & 0 & 0 \\ 23 & 86 & 5 & 6\end{array}$

\section{DISCUSSION}

The data indicate that conversion of diploid and metastable stocks of Dictyostelium discoideum to the stable haplophase occurs during growth and, in the absence of clonal re-isolation, there is a progressive increase in the proportion of stable haploids. Under certain cultural conditions this process is facilitated by selective advantages enjoyed by the haploid segregants, possibly because of the uncovering of recessive alleles affecting growth rate.

The mechanisms of diploidization and haploidization in Dictyostelium discoideum are unknown at present. The existence of heterozygotic strains like $\mathrm{H}-1$ forces the conclusion that at least some diploids arise by cell fusion and karyogamy. Several mechanisms of haploidization can be envisaged. An orthodox meiotic reduction may occur such as proposed by Wilson (1953) and Wilson \& Ross (1955). Cytological support for this process is suggestive but equivocal. Vegetative reduction division by means of tripolar and tetrapolar mitoses is a second possibility. Experimental support for this includes the tri- and tetrapolar mitotic figures encountered in haploidizing cultures (see Pl. 1) and observations of ternary and quaternary fissions by time-lapse cinematography (Sussman, Sussman \& Ennis, 1960). A third 


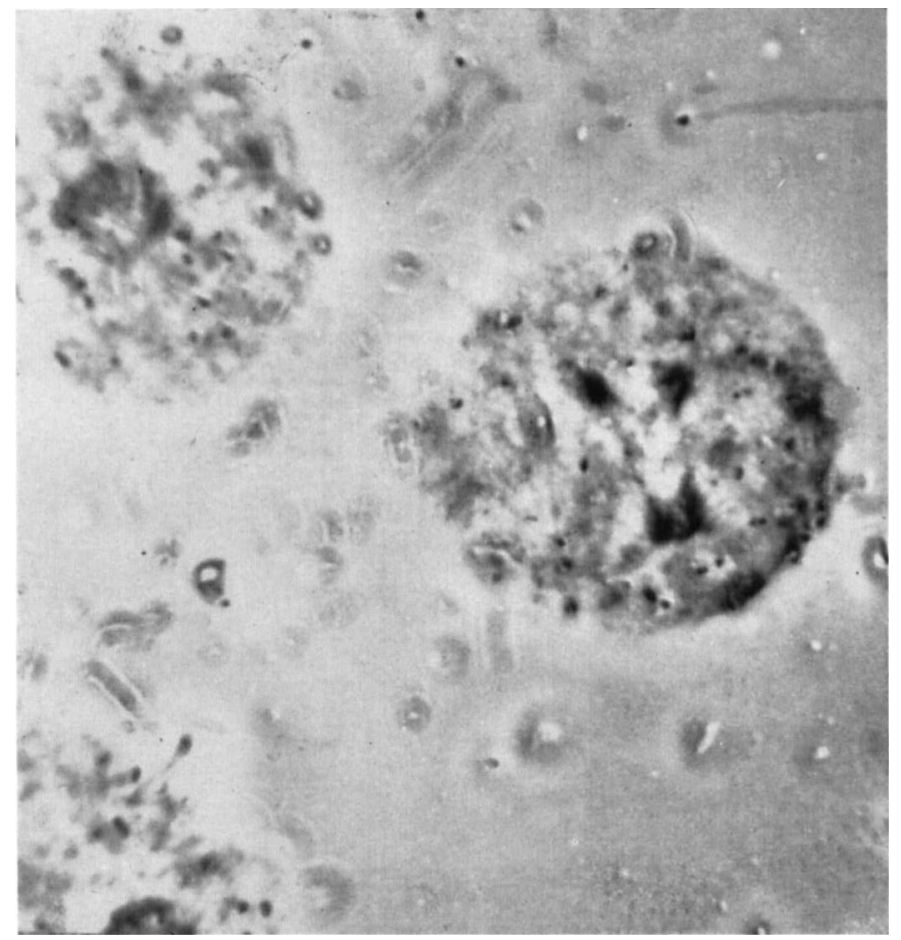


possibility is a piecemeal loss of chromosomes from the diploid by non-disjunction or other means. In this case the haplophase would be preceded by transitory aneuploidy lasting over several generations. The latter has been observed in at least one fungal group, Aspergillus (Käfer, 1960). At present it is impossible to choose among these hypotheses.

Support by grants from the National Institutes of Health (C-4057) and the National Science Foundation (G-12900) is gratefully acknowledged.

\section{REFERENCES}

Gerisch, G. (1960). Ein Submerskulturverfahren für Dictyostelium discoideum. Naturwissenschaften, 46, 654 .

KäFER, E. (1960). High frequency of spontaneous and induced segregation in Aspergillus nidulans. Nature, Lond. 186, 619.

Ross, I. K. (1960). Studies on diploid strains of D. discoideum. Amer. J. Bot. 47, 54 .

Sussman, M. (1951). Origin of cellular heterogeneity in the slime molds, Dictyosteliaceae. J. exp. Zool. 118, 407.

Sussman, M., Sussman, R. R. \& Ennis, H. L. (1960). Appearance and inheritance of the I-cell phenotype in D. discoideum. Devel. Biol. 2, 367.

Sussman, M. (1961 a). Cultivation and serial transfer of Dictyostelium discoideum in liquid nutrient medium. J. gen. Microbiol. 25, 375.

Sussman, M. (1961 b). Consequences of an act of cellular differentiation in the cellular slime molds. Purdue Univ. Symp. on Growth. Basic Books, Publ. p. 221.

Sussman, M. \& Sussman, R. R. (1962). Ploidal inheritance in Dictyostelium discoideum I. J. gen. Microbiol. 28, 417 .

Sussman, R. R. (1961). A method for staining chromosomes of D. discoideum. Exp. Cell Res. 24, 154.

Wilson, C. M. (1953). Cytological study of the life cycle of Dictyostelium. Amer. J. Bot. 40, 714.

WILson, C. M. \& Ross, I. K. (1955). Meiosis in the Myxomycetes. Amer. J. Bot. 42, 743.

Wirson, C. M. \& Ross, I. K. (1957). Further cytological studies on the Acrasiales. Amer. J. Bot. 44, 345.

\section{EXPLANATION OF PLATE}

Photomicrograph of tripolar mitotic (telophase) figure $(\times 2000)$. Aceto-orcein stain after Carnoy fixation and acid hydrolysis. 